\title{
MS33-04 | The Crystalline Sponge Method: Pitfalls, Challenges and Solutions
}

de Gelder, Rene (Radboud University, Nijmegen); de Poel, Wester (Radboud University, Nijmegen); Tinnemans, Paul (Radboud University, Nijmegen); Duchateau, Alexander L.L. (DSM Biotechnology Center, Delft); Honing, Maarten (Maastricht University, Maastricht); Rutjes, Floris P.J.T. (Radboud University, Nijmegen); Vlieg, Elias (Radboud University, Nijmegen)

The crystalline sponge (CS) method promises the elucidation of the (absolute) structure of molecules using singlecrystal X-ray diffraction and eliminates the need for crystals of the target compound. The target compound is absorbed into a porous host crystal and by applying single-crystal X-ray diffraction to the host-guest complex, structure elucidation of the target compound becomes possible. There are, however, important factors that limit the successful application of the CS method: there is no universal sponge that can host every possible target compound, ordering of guest molecules inside the host framework is rather unpredictable, instability of the crystalline sponges limits the range of molecules that can be investigated and guest exchange may affect the quality of the host-guest crystals.

In this contribution we present our experience with the CS method and attempts to solve the problems mentioned. Powder X-ray diffraction provides a direct view on incorporation of ordered guest molecules and was used to determine the optimal and/or minimal soaking time. Enantiomeric pairs were found to order inside the channels of the host framework when a racemic guest instead of an enantiopure compound is used. Stable MOF hosts based on f-block metals make application of the CS method to a wider array of guests and solvents possible. Cooperative ordering, selective ordering, and joint disorder were observed, dependent on the specific combinations and ratios of guest compounds. Our results show that the CS method still needs a lot of development before it will become generally applicable and accepted by the crystallographic community. 